\title{
Modelo Matemático de la Nucleación Electroquímica con Ondas de Corriente Pulsante
}

\author{
Jarol E. Molina ${ }^{(1)}$ y Bibian A. Hoyos ${ }^{(2)^{*}}$ \\ (1) Universidad Nacional de Colombia, Fac. de Minas, Of. M4-214, Carrera 80, No. 65-223, \\ Medellín- Colombia (jemolina@unal.edu.co) \\ (2) Universidad Nacional de Colombia, Escuela de Procesos y Energía, Apdo. Aéreo 1027, \\ Medellín-Colombia (bahoyos@unal.edu.co)
}

* autor a quien debe ser dirigida la correspondencia

\section{Resumen}

Se presenta un modelo matemático para describir el sobrepotencial de concentración, la velocidad de nucleación y el tamaño de núcleos formados sobre sustratos metálicos, cuando se emplean cuatro tipos de ondas de corriente pulsante: rectangular, rampa ascendente, rampa descendente y triangular. Para el desarrollo del modelo, se considera que la etapa que controla el proceso es la difusión de especies en la capa limite. El modelo predice que, cuando se emplean ondas con igual corriente promedio e igual tiempo de aplicación, la rampa descendente produce tiempos de transición más cortos, con mayor aumento en el sobrepotencial de concentración, lo que lleva a mayores velocidades de nucleación con tamaños de núcleos más pequeños. Para el caso de ondas con igual corriente pico y promedio, las ondas rectangular y descendente presentan prácticamente el mismo tiempo de transición, con velocidades de nucleación y tamaño de los depósitos equivalentes. El modelo desarrollado, constituye una herramienta ágil y flexible para la determinación del tamaño y la velocidad de formación de núcleos de electrodepositos y, puede ser extendida para estudiar otro tipo de ondas de corriente.

\section{Mathematical Model for the Electrochemical Nucleation with Pulse Current Waveforms}

\begin{abstract}
A mathematical model has been established for the description of concentration overpotential, nucleation rate and nucleus size formed on metallic substrates when four types of pulse current waveforms are used: rectangular, ramp-down, ramp-up and triangular. The mathematical model was developed considering that the species diffusion in the limit layer is the rate determining step of the process. The model predicts that when using wave current with the same current average and the same time of application, the ramp-down current produces the shortest transition time, with the highest rise of overpotential concentration, this drives to high nucleation rates with small nucleus size. For the case of current wave forms with the same pick and average current, the rectangular and ramp-down waves show practically the same transition time, with equivalent nucleation rate and deposit size.
\end{abstract}

Keywords: electrochemical nucleation, pulse current, mathematical model, overpotential, electrocrystallization 


\section{INTRODUCCIÓN}

En la actualidad los procesos de electrocristalización, mediante el empleo de ondas de corriente pulsante, han tomado una gran importancia tecnológica: La comprensión de los fenómenos que ocurren en el proceso de nucleación y crecimiento (Grujicic y Pesic, 2002; Milchev y Heerman, 2003), los estudios para determinar la composición y las propiedades electroquímicas del electrolito adecuadas para depositar múltiples metales (Milchev et al., 2004; Hansal et al., 2006) y, el análisis y desarrollo de técnicas adecuadas para medir las velocidades de nucleación (Milchev y Michailova, 2000), son algunos de los estudios que se llevan a cabo en la actualidad. El interés creciente por esta técnica nace de las ventajas económicas que representa, ya que se pueden mejorar características superficiales como la dureza y la resistencia a la abrasión del material (entre otras), con disminuciones importantes en los costos de fabricación por concepto de ahorro de reactivos utilizados y disminución de la potencia eléctrica (Wong et al., 2001a). Adicionalmente, las ondas de corriente pulsante se han empleado para obtener depósitos de níquel con menor aspereza (Wong et al., 2001b), para la producción de nanocristales, para disminuir la corrosión y aumentar la pureza de los depósitos (Youssef et al, 2004) y en el campo de la electrónica (Georgianou y Veyret 2002), entre otros.

Recientemente se han publicado simulaciones Monte Carlo del proceso de electrodepositación en condiciones de equilibrio y la extensión de estos resultados para condiciones dinámicas (Milchev et al., 2002). Estas simulaciones muestran que para aglomerados de tamaño grande (30 átomos o más), la ecuación de Young provee una buena aproximación. Sin embargo, para aglomerados muy pequeños las ecuaciones macroscópicas no son adecuadas y conceptos como el ángulo de contacto pierden sentido.

Por otra parte, se han publicado algunos modelos matemáticos que prentenden describir el proceso de electrodepositación mediante ecuaciones de transporte (Wong et al., 2001a, 2001b, 2001c, Georgiadou y Veret 2002), pero en algunos casos las soluciones planteadas no son confiables, puesto que presentan problemas de continuidad y en otros casos los modelos son potenciostáticos o se solucionan numéricamente, lo que conlleva a soluciones aproximadas y en ocasiones a largos tiempos de calculo.

En este trabajo, se presenta un modelo analítico completo, que describe la dinámica del proceso de electrodepositación, incluyendo la velocidad de nucleación tri-dimensional y el tamaño de grano de los electrodepósitos formados con cuatro tipos de ondas de corriente pulsante: rectangular, rampa ascendente, rampa descendente y triangular. Este modelo permite analizar el cambio en el tiempo de la concentración, el sobrepotencial, la velocidad de nucleación y, como éstas afectan la distribución de los tamaños de núcleo.

\section{MODELO MATEMÁTICO}

La formulación del modelo está directamente determinada por la forma de las ondas de corriente que se aplican al sistema, puesto que la corriente afecta la sobresaturación electroquímica y ésta se relaciona de forma directa con la velocidad de nucleación.

Los diferentes tipos de onda utilizados en este estudio se muestran en la figura 1. La descripción matemática de estas ondas se realiza empleando la función impulso unitario definida por:

$S_{k}(t)=\left\{\begin{array}{l}0 \rightarrow t<k \\ 1 \rightarrow t \geq k\end{array}\right.$

Las funciones matemáticas que representan el comportamiento de la corriente para las ondas rectangular ( $r$ ), rampa ascendente (ra), rampa descendente (rd) y triangular (tr), se pueden escribir mediante las ecuaciones 2 a 5, respectivamente: 
$i_{r}(t)=i_{p}\left[S_{w}(t)-S_{k}(t)\right]$

$i_{r a}(t)=\frac{i_{p}}{a}(t-w)\left[S_{w}(t)-S_{k}\right]$

$i_{r d}(t)=\frac{i_{p}}{a}(k-t)\left[S_{w}(t)-S_{k}(t)\right]$

$i_{t r}(t)=i_{p}\left[\frac{(t-w)}{a}\left[S_{w}(t)-S_{k}(t)\right]+\frac{(h-t)}{b}\left[S_{k}(t)-S_{h}(t)\right]\right]$

Con $w=N t, k=w+a$ y $h=k+b$ en las cuales $N(=0,1,2, \ldots, \mathrm{n})$ es el número de períodos completados, $\tau$ el período de la onda e $\mathrm{i}_{\mathrm{p}}$ es la corriente pico.
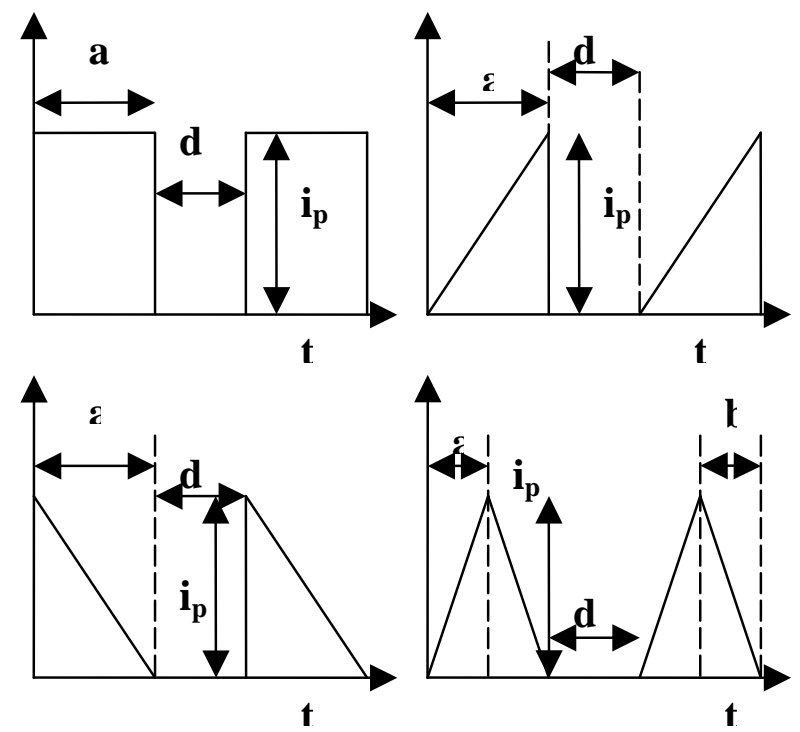

Fig. 1: Tipos de onda utilizados en el modelo

El proceso de electro-cristalización implica necesariamente la formación de una nueva fase. La energía requerida en la formación de ésta nueva fase es una barrera energética que está relacionada con la acumulación de átomos necesarios para formar un aglomerado de átomos estables. La formulación general de la energía libre de formación de un aglomerado está dada por:

$$
\begin{aligned}
& \Delta \widetilde{G}(n)=-n \Delta \widetilde{\mu}+\widetilde{\Phi}(n) \\
& \widetilde{\Phi}(n)=\widetilde{G}(n)-n \widetilde{\mu}_{\infty}^{M}(\phi)
\end{aligned}
$$

El término positivo en la ecuación (6) representa el trabajo empleado en la formación de la nueva fase, el termino negativo es el trabajo ganado por transferir $n$ átomos de un estado de alta energía en la solución a uno de baja energía en el depósito ya que $\widetilde{\mu}_{\infty}^{\text {sol }}\left(a_{\infty}^{\text {sol }}\right)>\widetilde{\mu}_{\infty}^{M}(\phi)$.

Si se considera que el núcleo que se forma sobre el sustrato tiene la forma de una gota, la ecuación (7) toma la forma:

$$
\widetilde{\Phi}(n)=\left[S(n)-S_{i}(n) \cos \gamma_{0}\right] \sigma
$$


Donde $S$ y $S_{i}$ representan el área del sustrato cubierta por el nuevo aglomerado y el área superficial del aglomerado, respectivamente. $\sigma$ corresponde a la energía libre específica interfacial y $\gamma_{0}$ es el ángulo de contacto entre la superficie y el aglomerado. Rescribiendo la ecuación (6) con la ayuda de la ecuación anterior (8) se puede llegar a:

$$
\begin{aligned}
& \Delta \widetilde{G}\left(n_{c}\right)=\frac{1}{2} n_{c} \Delta \widetilde{\mu} \\
& n_{c}=\frac{32 \pi \sigma^{3} v_{M}^{2}}{3 \Delta \tilde{\mu}^{3}} \xi\left(\gamma_{0}\right)
\end{aligned}
$$

$\Delta \tilde{\mu}=z_{+} e \eta$

$n_{c}$ representa el tamaño de núcleo crítico, definido como el aglomerado de la nueva fase que está en equilibrio inestable con la fase madre sobresaturada y,

$\eta=E_{\infty}-E$

$\eta$ es el sobrepotencial, que para el caso de un proceso controlado por la transferencia de masa, se puede expresar como:

$\eta=\frac{R T}{z F} \ln \left(\frac{C(t, 0)}{C_{0}}\right)$

$\xi\left(\gamma_{0}\right)$ Es una función que depende de la forma del aglomerado sobre el sustrato y tiene la siguiente estructura:

$\xi\left(\gamma_{0}\right)=1 / 2-(3 / 4) \cos \left(\gamma_{0}\right)+(1 / 4) \cos ^{3}\left(\gamma_{0}\right)$

La velocidad de nucleación se puede expresar como:

$V_{\text {nuc }}=\frac{i(t)}{Z_{+} e n_{c}}$

Como se puede ver de la ecuación (13), es necesario conocer la concentración en la superficie del electrodo para así calcular el sobrepontencial de concentración y hallar tanto el tamaño de los núcleos (Ec. 10) como su velocidad de formación (Ec. 15).

Si se considera que el sustrato es una superficie plana equipotencial, sin efectos de borde y que la etapa controlante del proceso es la difusión de especies en la capa limite, se puede describir la concentración en la superficie del sustrato mediante la segunda ley de Fick, es decir: 
$\frac{\partial C}{\partial t}=D \frac{\partial^{2} C}{\partial x^{2}}$

Con las siguientes condiciones de frontera e inicial:

$$
\begin{aligned}
& C(0, x)=C_{0} \\
& C(t, \infty)=C_{0} \\
& \frac{\partial C}{\partial x} \|_{x=0}=\frac{i(t)}{z F D}
\end{aligned}
$$

La ecuación diferencial parcial (Ec. 16) se puede resolver mediante transformada de Laplace (Rice y Do, 1995; Jonson y Jeffreys, 1969). En la tabla 1 se presenta la solución del perfil de concentraciones y del sobrepotencial para cada tipo de onda.

\section{RESULTADOS Y DISCUSIÓN}

En las figuras 2 y 3 se presenta el perfil de concentraciones que se obtiene para diferentes tipos de ondas de corriente. En el caso de la figura 2, las ondas aplicadas tienen la misma corriente promedio y el mismo tiempo de aplicación. Allí se observa que inicialmente la concentración en la superficie del electrodo cae más rápidamente cuando se aplica la rampa descendente. También se observa que cuando la corriente alcanza valores bajos, al final de la aplicación de las ondas triangular y descendente, el efecto de la difusión de las especies hacia el sustrato se hace importante, lo que produce un aumento en la concentración. Por otra parte, con la rampa ascendente, la concentración cae continuamente produciendo el mayor cambio de concentración de todas las ondas empleadas.

La figura 3 muestra el perfil de concentraciones para ondas con igual corriente promedio e igual corriente pico (la onda rectangular se aplica la mitad del tiempo que las ondas triangulares). Se observa en este caso que la onda rectangular produce el mayor cambio de concentración mientras dura su aplicación y que para el final del tiempo de aplicación de las ondas, la rampa ascendente es la que produce el segundo mayor cambio en la concentración.

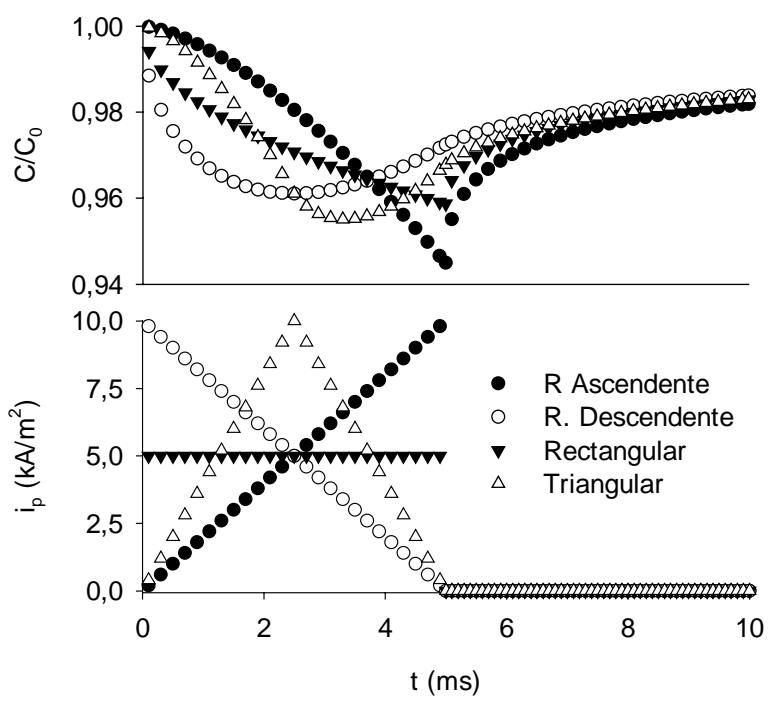

Fig. 2: Perfil de concentración en la superficie para ondas con igual corriente promedio e igual tiempo de aplicación (5 ms). ( $\left.\mathrm{D}=4.7 * 10^{-10} \mathrm{~m}^{2} / \mathrm{s}\right)$ : 


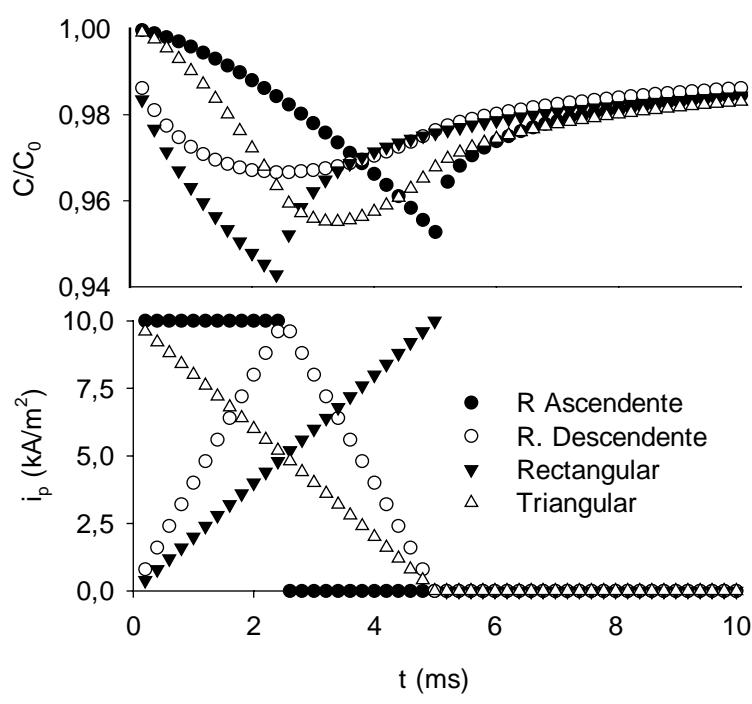

Fig. 3: Perfil de concentración en la superficie para ondas con igual corriente promedio $\left(2.5 \mathrm{kA} / \mathrm{m}^{2}\right)$ e igual $i_{p}\left(10 \mathrm{kA} / \mathrm{m}^{2}\right)\left(\right.$ con $\left.\mathrm{D}=4.7^{\star} 10^{-10} \mathrm{~m}^{2} / \mathrm{s}\right)$

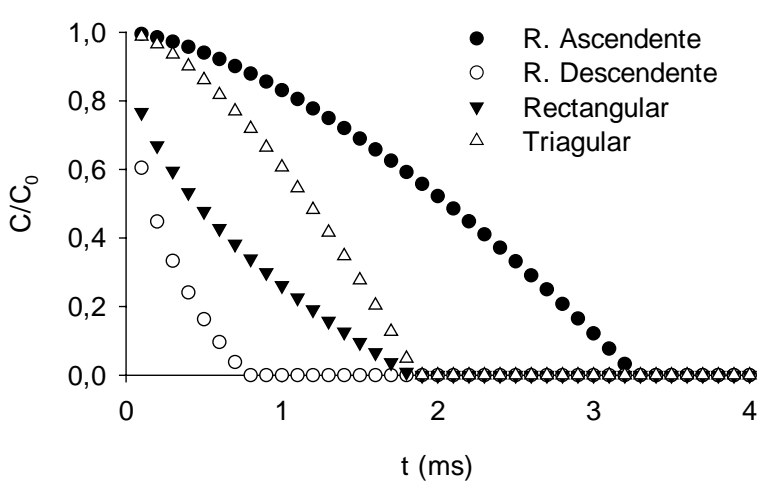

Fig. 4: Tiempos de transición para las ondas con igual corriente promedio $\left(100 \mathrm{kA} / \mathrm{m}^{2}\right)$ e igual tiempo de aplicación (5 ms). ( $\left.\mathrm{D}=4.7 * 10^{-10} \mathrm{~m}^{2} / \mathrm{s}\right)$

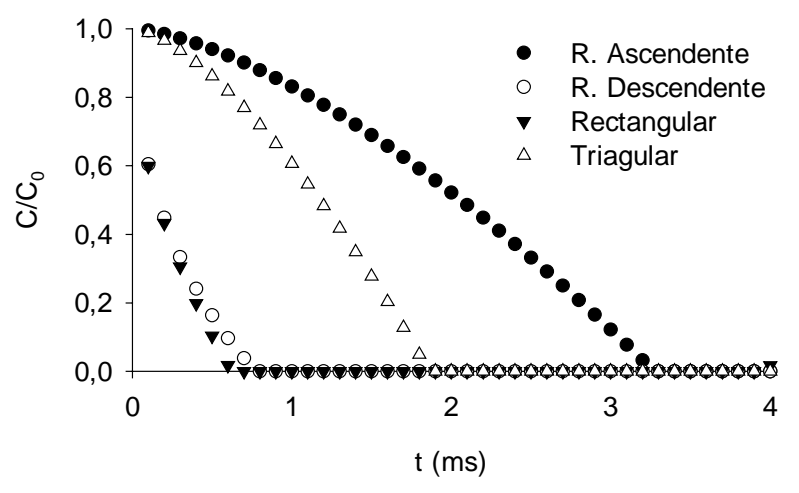

Fig. 5: Tiempos de transición para las ondas con igual corriente promedio $\left(100 \mathrm{kA} / \mathrm{m}^{2}\right)$ e igual $\mathrm{i}_{\mathrm{p}}$ (con $\mathrm{D}=4.7 * 10^{-10} \mathrm{~m}^{2} / \mathrm{s}$ ) 
Tabla 1: Perfiles de concentración para la onda rectangular, rampa ascendente, rampa descendente y triangular

Rectangular

$C(t, 0)=C_{0}-\frac{i_{p}}{z F \sqrt{D}}\left[\frac{S_{w}(t) \sqrt{t-w}}{\Gamma(3 / 2)}-\frac{S_{k}(t) \sqrt{t-k}}{\Gamma(3 / 2)}\right]$

$$
\eta_{r}=\frac{R T}{z F} \ln \left(1-\frac{i_{p}}{z F C_{0} \sqrt{D}}\left[\frac{S_{w}(t) \sqrt{t-w}}{\Gamma(3 / 2)}-\frac{S_{k}(t) \sqrt{t-k}}{\Gamma(3 / 2)}\right]\right)
$$

Rampa Ascendente

$C(t, 0)=C_{0}-\frac{i_{p}}{z F \sqrt{D} a}\left[\frac{(t-w)^{3 / 2}}{\Gamma(5 / 2)} S_{w}(t)-\frac{(t-k)^{3 / 2}}{\Gamma(5 / 2)} S_{k}(t)-a \frac{(t-k)^{1 / 2}}{\Gamma(3 / 2)}\right] \quad \eta_{r a}=\frac{R T}{z F} \ln \left(1-\frac{i_{p}}{z F C_{0} \sqrt{D} a}\left[\frac{(t-w)^{3 / 2}}{\Gamma(5 / 2)} S_{w}(t)-\frac{(t-k)^{3 / 2}}{\Gamma(5 / 2)} S_{k}(t)-a \frac{(t-k)^{1 / 2}}{\Gamma(3 / 2)}\right]\right)$

\section{Rampa Descendente}

$C(t, 0)=C_{0}-\frac{i_{p}}{z F \sqrt{D} a}\left[a \frac{(t-w)^{1 / 2}}{\Gamma(3 / 2)} S_{w}(t)-\frac{(t-w)^{3 / 2}}{\Gamma(5 / 2)} S_{w}(t)+\frac{(t-k)^{3 / 2}}{\Gamma(5 / 2)}\right] \quad \eta_{r d}=\frac{R T}{z F} \ln \left(1-\frac{i_{p}}{z F C_{0} \sqrt{D} a}\left[a \frac{(t-w)^{1 / 2}}{\Gamma(3 / 2)} S_{w}(t)-\frac{(t-w)^{3 / 2}}{\Gamma(5 / 2)} S_{w}(t)+\frac{(t-k)^{3 / 2}}{\Gamma(5 / 2)}\right]\right)$

Triangular

$$
\begin{gathered}
C(t, 0)=C_{0}-\frac{i_{p}}{z F \sqrt{D}}\left[\frac{1}{a}\left(\frac{(t-w)^{3 / 2}}{\Gamma(5 / 2)} S_{w}(t)-\frac{(t-k)^{3 / 2}}{\Gamma(5 / 2)} S_{k}(t)-a \frac{(t-k)^{1 / 2}}{\Gamma(3 / 2)} S_{k}\right)-\frac{1}{b}\left(\frac{(t-k)^{3 / 2}}{\Gamma(5 / 2)} S_{k}(t)-b \frac{(t-k)^{1 / 2}}{\Gamma(3 / 2)} S_{k}(t)-\frac{(t-h)^{3 / 2}}{\Gamma(5 / 2)} S_{h}(t)\right)\right] \\
\eta_{t r}=\frac{R T}{z F} \ln \left(1-\frac{i_{p}}{z F C_{0} \sqrt{D}}\left[\frac{1}{a}\left(\frac{(t-w)^{3 / 2}}{\Gamma(5 / 2)} S_{w}(t)-\frac{(t-k)^{3 / 2}}{\Gamma(5 / 2)} S_{k}(t)-a \frac{(t-k)^{1 / 2}}{\Gamma(3 / 2)} S_{k}\right)-\frac{1}{b}\left(\frac{(t-k)^{3 / 2}}{\Gamma(5 / 2)} S_{k}(t)-b \frac{(t-k)^{1 / 2}}{\Gamma(3 / 2)} S_{k}(t)-\frac{(t-h)^{3 / 2}}{\Gamma(5 / 2)} S_{h}(t)\right)\right]\right)
\end{gathered}
$$

En la figura 4, se observa que para ondas con igual corriente promedio y e igual tiempo de aplicación, el menor tiempo de transición se obtiene cuando se emplea la rampa descendente. Para el caso de ondas con igual corriente pico y promedio (figura 5), la onda rectangular presenta un tiempo de transición levemente inferior a la rampa descendente. Adicionalmente, se observa que en este caso los tiempos de transición de estas ondas es un orden de magnitud menor que los correspondientes a las ondas triangular y ascendente.

Cuando se alcanza el tiempo de transición durante la aplicación de una onda de corriente, el sobrepotencial de concentración se aumenta significativamente, lo cual incrementa la velocidad de nucleación y se producen depósitos con tamaños de grano más pequeños (como se puede ver en las Figuras 6 y 7 ). 


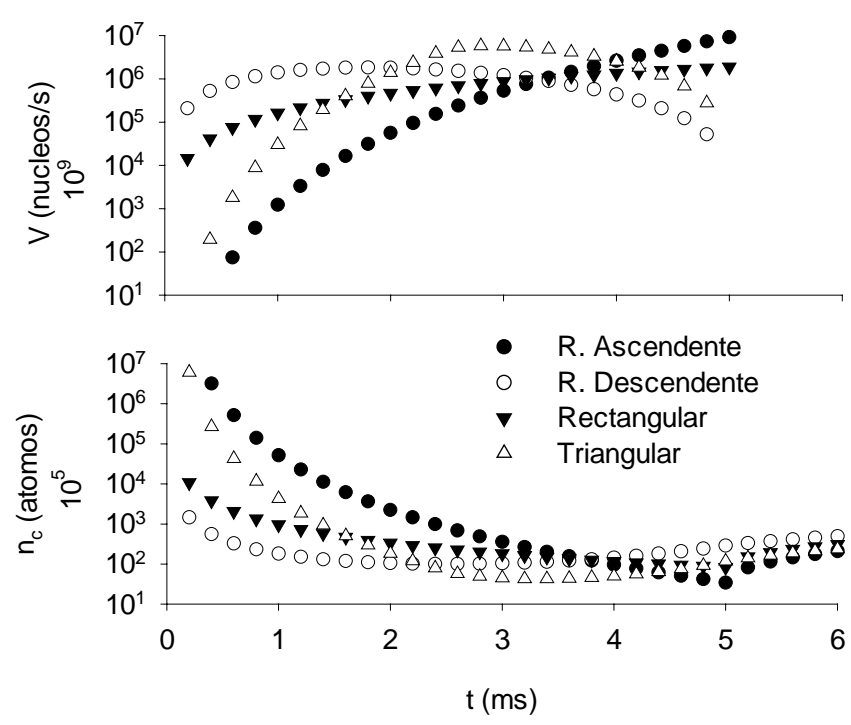

Fig. 6: Velocidad de nucleación y tamaño critico de núcleo para ondas con igual corriente promedio $\left(2.5 \mathrm{kA} / \mathrm{m}^{2}\right)$ e igual tiempo de aplicación $(5 \mathrm{~ms})$. (con $\left.\mathrm{D}=4.7^{\star} 10^{-10} \mathrm{~m}^{2} / \mathrm{s}\right)$.

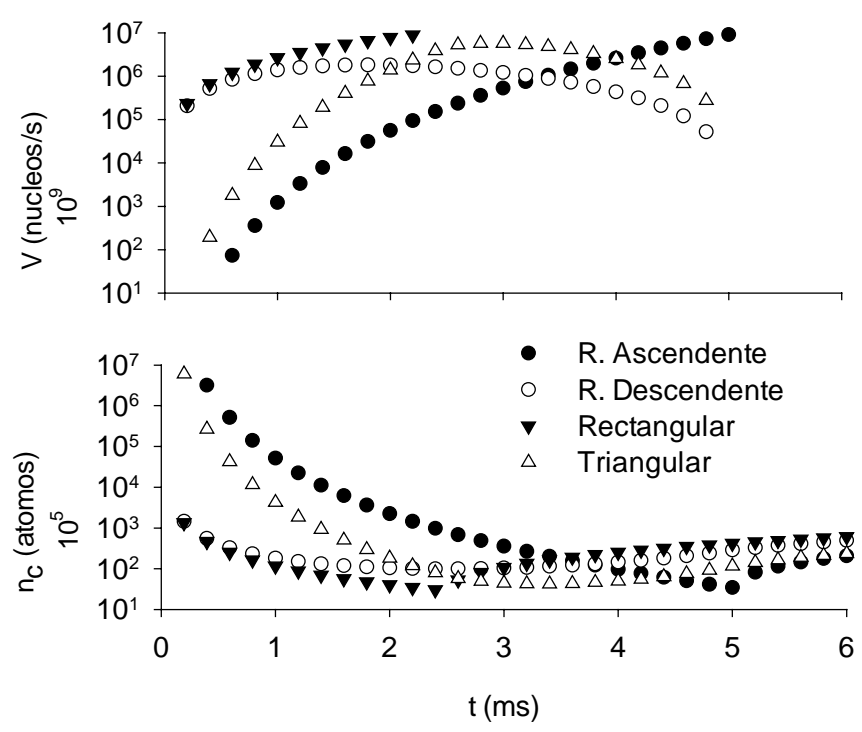

Fig. 7:. Velocidad de nucleación y tamaño critico de núcleo para ondas con igual corriente promedio $\left(2.5 \mathrm{kA} / \mathrm{m}^{2}\right)$ e igual $\mathrm{i}_{\mathrm{p}}\left(10 \mathrm{kA} / \mathrm{m}^{2}\right)\left(\right.$ con $\left.\mathrm{D}=4.7^{\star} 10^{-10} \mathrm{~m}^{2} / \mathrm{s}\right)$.

En la figura 6 se observa que, para el primer tipo de ondas consideradas, los tamaños de núcleos más pequeños y las mayores velocidades de nucleación se obtienen con la rampa descendente, seguida por la onda rectangular. La rampa ascendente y la onda triangular producen tamaños de núcleos mucho más grandes con una velocidad de formación mucho menor.

Para el caso de ondas con igual corriente pico e igual corriente promedio (figura 7), las ondas ascendente y triangular siguen produciendo tamaños de núcleos más grandes que la rampa descendente y la onda rectangular, pero la diferencia entre estas dos últimas ya no es significativa. La rampa descendente y la onda rectangular producirán depósitos con menor tamaño y mayor dureza (como era de esperarse debido a los tiempos de transición menores). 


\section{CONCLUSIONES}

El modelo de nucleación desarrollado muestra que se puede comparar el efecto de la aplicación de diferentes formas de onda de corriente sobre la velocidad de formación y el tamaño de los núcleos de los depósitos formados. El modelo predice que cuando se comparan ondas con igual corriente promedio e igual tiempo de aplicación, la rampa descendente produce tiempos de transición más cortos, con mayor aumento en el sobrepotencial de concentración lo que lleva a mayores velocidades de nucleación con tamaños de núcleo más pequeños.

Cuando se comparan ondas con igual corriente pico y promedio, las ondas rectangular y descendente producen prácticamente el mismo tiempo de transición, lo que produce velocidades de nucleación y tamaño de los depósitos prácticamente equivalentes.

El modelo desarrollado en este trabajo constituye una herramienta ágil y flexible para la determinación del tamaño y la velocidad de formación de núcleos de electrodepositos y puede ser fácilmente extendida para estudiar otro tipo de ondas de corriente.

Este modelo se puede emplear también para estudiar el efecto del porcentaje de ciclo de trabajo y de la frecuencia de la onda cuando se emplean ciclos permanentes de corriente, lo cual será motivo de estudio en otro trabajo.

\section{AGRADECIMIENTOS}

Este trabajo se pudo llevar a cabo gracias a la financiación de COLCIENCIAS (código: 1118-0817212) y de la dirección de investigación de Universidad Nacional de Colombia-Medellín (DIME)

\section{REFERENCIAS}

Georgiadou, M. y D. Veyret; Modeling of Transient Electrochemical Systems Involving Moving Boundaries, J. Electrochem. Soc.: 149 (6), C324-C330 (2002).

Grujicic, D. y B. Pesic; Electrodeposition of copper: the nucleation mechanisms, Electrochimica Acta: 41, $2901-2912$ (2002).

Hansal, W.E.G. y otros cuatro autores; Pulse plating of Ni-Co alloys: Depotion Kinetics of Watts, Sulfamate and choride electrolytes, Electrochimica Acta: 52, 1145-1151, (2006).

Jonson, V.G. y G.V. Jeffreys; Métodos Matemáticos en Ingeniería Química, Alambra, Madrid, España (1969).

Milchev, A. y E. Michailova; Studies of electrochemical nucleation by means of standard and modified pulse potentiostatic techniques, Electrochem. Comm.: 2, 15 - 17 (2000).

Milchev, A., A. Milchev y K. Binder; Nanodroplets on a solid plane: wetting and spreading in a Monte Carlo simulation, Computer Phys. Commu.: 146, 38-53 (2002)

Milchev, A. y L. Heerman; Electrochemical nucleation and growth of nano- and microparticles: some theoretical and experimental aspects, Electrochim. Acta: 48, 2903-2913 (2003).

Milchev, A., E. Michailova y T. Zapryanova; Initial stages of electrochemical alloy formation: size and composition of critical nuclei, Electrochem. Comm.: 6, 713-718 (2004).

Rice, R.G. y D.D. Do; Appied Mathematics and Modeling for Chemical Engineers, John Wiley \& Sons, New York, USA (1995).

Wong, K.P., K.C. Chan y T.M. Yue; A study of hardness and grain size in pulse current electroforming of nickel using different shaped waveforms, J. Appl. Electrochem.: 31, 25-34 (2001a). 
Wong, K.P., K.C. Chan y T.M. Yue; Modelling the effect of complex waveform on surface finishing in pulse current electroforming of nickel, Surface and Coating Tech.: 135, 91-97 (2001b).

Wong, K.P., K.C. Chan y T.M. Yue; Modelling of electrocrytallization for pulse current electroforming of nickel, Appl. Surf. Sci.: 178, 178-189 (2001c).

Youssef, K., C.C. Koch y P.S. Fedkiw; Influence of Additives and Pulse Electodeposition Parameters on Production of Nanocrystalline Zinc for Zinc Coloride, J. Electrochem. Soc.: 151 (2), C103-C111 (2004). 